\title{
An Improved Approximation for the Gaussian $Q$-Function
}

\author{
George K. Karagiannidis, Senior Member, IEEE, and Athanasios S. Lioumpas Student Member, IEEE
}

\begin{abstract}
We present a novel, simple and tight approximation for the Gaussian $Q$-function and its integer powers. Compared to other known closed-form approximations, an accuracy improvement is achieved over the whole range of positive arguments. The results can be efficiently applied in the evaluation of the symbol error probability (SEP) of digital modulations in the presence of additive white Gaussian noise (AWGN) and the average SEP (ASEP) over fading channels. As an example we evaluate in closed-form the ASEP of differentially encoded QPSK in Nakagami- $m$ fading.
\end{abstract}

Index Terms-Gaussian $Q$-function, Differentially encoded QPSK, Nakagami- $m$ fading.

\section{INTRODUCTION}

$\mathbf{T}$ HE Gaussian $Q$-function, the directly related error function $\operatorname{erf}(\cdot)$ and its complement function $\operatorname{erfc}(\cdot)$ are of great importance in communication theory problems where the noise is often characterized by the Gaussian distribution [1]. Although, efficient numerical methods and infinite series have been proposed for the calculation of the $Q$-function [2], [3], no exact and simple expression is known, appropriate for mathematical manipulations. Towards this concept, several approximations have been derived in [4], [5]. In the former, two approximations are presented; the first is highly accurate but its mathematical form is complicated in order to be useful for mathematical manipulations, and the second is simple but with insufficient accuracy. In [5], a useful and simple approximation that involves the sum of two exponential functions was presented and the results were applied to the general problem of evaluating the average symbol error probability (ASEP) in fading channels. However, its accuracy vanishes for small arguments.

In this letter, we present a novel, simple and tighter approximation for the Gaussian $Q$-function and its integer powers, which can be applied in the evaluation of the ASEP of digital modulations in additive white Gaussian (AWGN) as well as fading channels. Contrary to other known closed-form approximations, a sufficient accuracy is guaranteed for all positive arguments. This fact is important while -as mentioned in [5]- bounds or approximations for the $Q$-function are not generally suitable for application to average error-probability

Manuscript received March 29, 2007. The associate editor coordinating the review of this paper and approving it for publication was Dr. Charalambos Charalambous. This work was performed within the framework of the Satellite Communications Network of Excellence (SatNEx) project (IST-507052) and its Phase-II, SatNEx-II (IST- 27393), funded by the European Commission (EC) under its FP6 program.

The authors are with the Electrical and Computer Engineering Department, Aristotle University of Thessaloniki, 54124 Thessaloniki, Greece (e-mail: \{geokarag, alioumpa\}@auth.gr).

Digital Object Identifier 10.1109/LCOMM.2007.070470. evaluation, because of the need to average over the signalto-noise ratio (SNR) between zero and infinity. By evaluating in closed-form the ASEP of the differentially encoded QPSK (DE-QPSK) in Nakagami- $m$ fading, we show that the approximation presented in this letter, can be efficiently applied for this type of problems, without loss in accuracy.

\section{AN IMPROVED APPROXIMATION FOR THE GAUSSIAN $Q$-FUNCTION}

The Gaussian $Q$-function is defined as

$$
Q(x)=\frac{1}{2} \operatorname{erfc}\left(\frac{x}{\sqrt{2}}\right)
$$

where

$$
\operatorname{erfc}(x)=\frac{2}{\sqrt{\pi}} \int_{x}^{\infty} e^{-t^{2}} d t
$$

is the complementary error function [6, (8.2.50)].

The erfc(x) can be represented by a continued fraction as [7, (7.1.14)]

$$
\operatorname{erfc}(x)=\frac{e^{-x^{2}}}{\sqrt{\pi}}\left(\frac{1}{x+} \frac{1 / 2}{x+} \frac{1}{x+} \frac{3 / 2}{x+} \frac{2}{x+} \ldots\right), \operatorname{Re}(x)>0
$$

A simple upper bound can be obtained by truncation at the second term as

$$
\operatorname{erfc}(x)<h(x)=\frac{e^{-x^{2}}}{\sqrt{\pi} x} .
$$

Compared to other well-known upper bounds, such as the Chernoff-Rubin [5], (4) is much worse for small arguments, but much tighter for large arguments. However, it is observed that by multiplying (4) with a monotonically increasing function of a specific form, a tightness in the small arguments region could be also achieved without losing the accuracy in the large arguments region. For this purpose, we utilize a function of the form

$$
g(x)=\frac{1-e^{-A x}}{B}
$$

where $A, B$ are constant real numbers. In Fig.1, we illustrate this concept by plotting $\operatorname{erfc}(x), h(x)$ and $g(x)$. Thus, the problem is to find the parameters $A$ and $B$ of the function

$$
f(x, A, B)=\frac{\left(1-e^{-A x}\right) e^{-x^{2}}}{B \sqrt{\pi} x} \approx \operatorname{erfc}(x)
$$

in order to minimize the integral of the absolute error in the range of values of interest, i.e.

$$
\{A, B\}=\underset{\{A, B\}}{\arg \min } \frac{1}{R} \int_{0}^{R}|f(x, A, B)-\operatorname{erfc}(x)| d x
$$




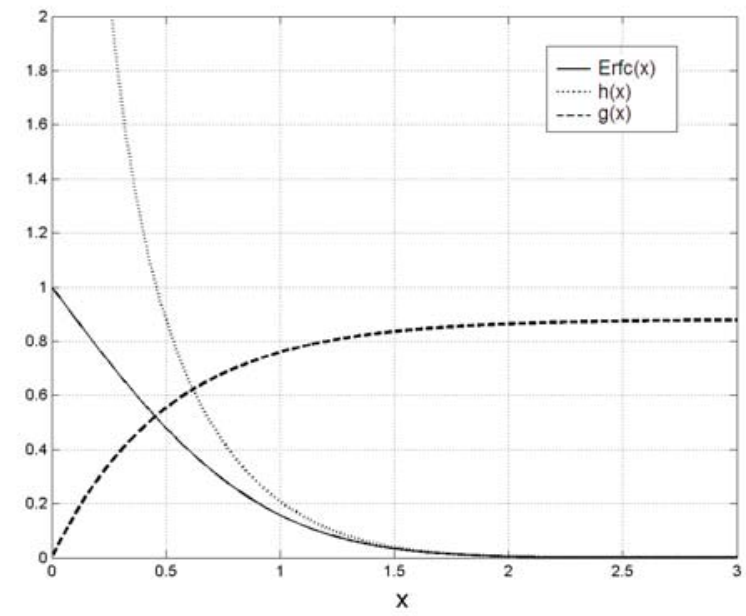

Fig. 1. An approximation of the erfc(x) can be obtained by multiplying $g(x)$ and $\mathrm{h}(\mathrm{x})$.

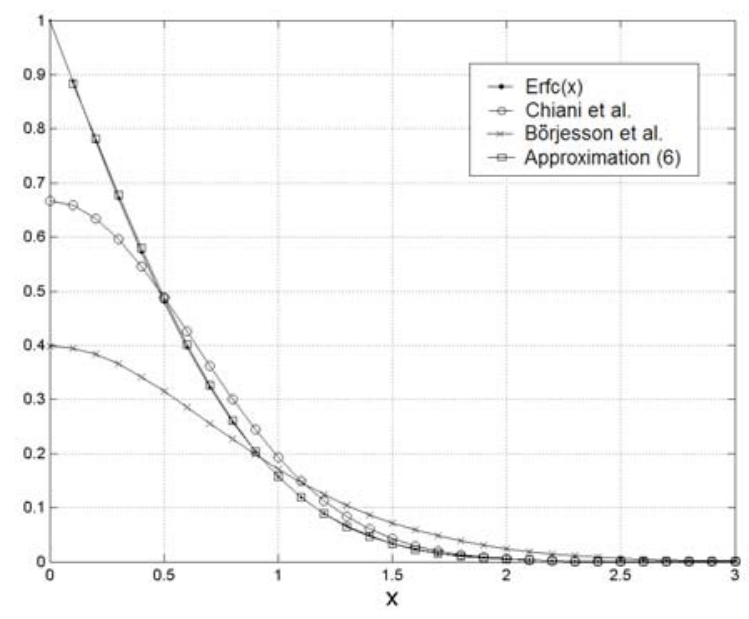

Fig. 2. Comparison between the erfc(x), (8), (9) and (6).

where $[0, R]$ is the arguments region of interest and $|\cdot|$ denotes absolute value. The optimal values of $A$ and $B$ depend on the range in which the integral of the absolute error is minimized. In other words, the accuracy of the approximation can be improved for a specific range of values, depending on the application. In this letter, the optimum values for $A, B$ were derived so that the $\operatorname{erfc}(x)$ is accurately approximated over the range of positive arguments, which is of practical interest in most communications systems applications. Thus, for $R=20$, it can be found numerically that the integral of the absolute error is minimized for $A=1.98$ and $B=1.135$. At this point, we should note that the proposed approximation is more accurate compared to $[5,(14)],[4,(9)]$ for values close to the origin with $\lim _{x \rightarrow 0} f(x, A, B)=0.984$ as well. Nonetheless, depending on the application, the accuracy at the origin could be further improved, by adding the constraint $\lim _{x \rightarrow 0} f(x, A, B)=1$ at the minimization problem given in (7); this, however, comes the cost of loss in accuracy for the rest of the region of $x$.

In Fig. 2 and Table I we compare the $\operatorname{erfc}(x)$ against the

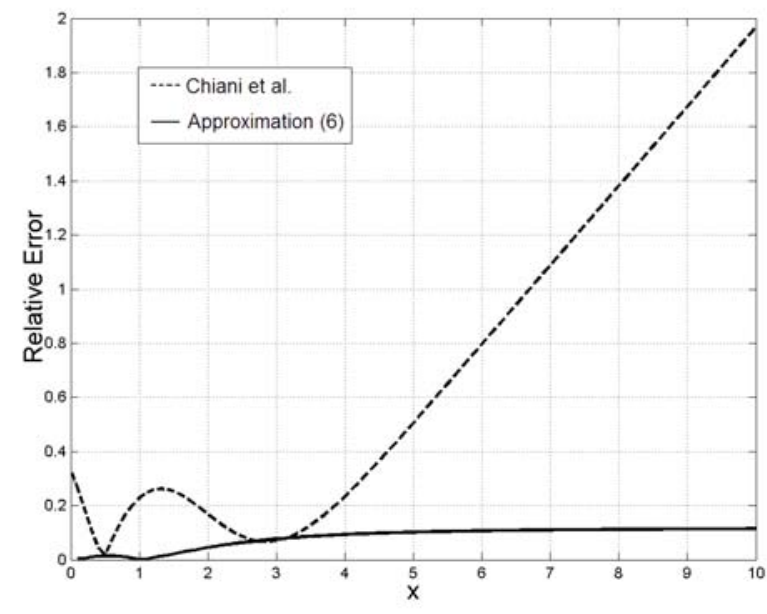

Fig. 3. The relative error of the approximations given by (6) and [5, Eq. 14].

TABLE I

COMPARISON BETWEEN ERFC(X), (8), (9) AND (6)

\begin{tabular}{|l|l|l|l|l|}
\hline \multicolumn{1}{|c|}{$\mathrm{x}$} & \multicolumn{1}{|c|}{$\operatorname{Erfc}(\mathrm{x})$} & Chiani et al. & Börjesson et al. & \multicolumn{1}{c|}{$(6)$} \\
\hline 0.1 & 0.887537 & 0.658386 & 0.394983 & 0.884027 \\
\hline 0.3 & 0.671373 & 0.595782 & 0.365303 & 0.678248 \\
\hline 0.5 & 0.479500 & 0.488066 & 0.314897 & 0.486562 \\
\hline 0.7 & 0.322199 & 0.362259 & 0.255808 & 0.326246 \\
\hline 0.9 & 0.203092 & 0.243941 & 0.197780 & 0.204350 \\
\hline 1 & 0.157299 & 0.193112 & 0.171099 & 0.157618 \\
\hline 2 & 0.0046777 & 0.0054665 & 0.0241455 & 0.0044654 \\
\hline 4 & $1.54173 \mathrm{e}-8$ & $1.90275 \mathrm{e}-8$ & 0.00003246 & $1.39798 \mathrm{e}-8$ \\
\hline 5 & $1.53746 \mathrm{e}-12$ & $2.31633 \mathrm{e}-12$ & $2.9157 \mathrm{e}-7$ & $1.38062 \mathrm{e}-12$ \\
\hline 10 & $2.08849 \mathrm{e}-45$ & $6.20013 \mathrm{e}-45$ & $7.65641 \mathrm{e}-24$ & $1.84919 \mathrm{e}-45$ \\
\hline
\end{tabular}

approximation given in $[5,(14)]$ as

$$
\operatorname{erfc}(x) \approx \frac{1}{6} e^{-x^{2}}+\frac{1}{2} e^{-\frac{4}{3} x^{2}},
$$

that given in $[4,(9)]$ as

$$
\operatorname{erfc}(x) \approx \frac{e^{-\frac{x^{2}}{2}}}{\sqrt{2 \pi} \sqrt{1+x^{2}}},
$$

and the proposed one given in (6). As it is observed, for a wide range of arguments and especially in the low arguments region, the approximation in (6) is more accurate than (8) and (9). In Fig. 3, we compare the relative error (RE) i.e.

$$
R E=\frac{|F(x)-\operatorname{erfc}(x)|}{\operatorname{erfc}(x)}
$$

of (8) and (6), where $F(x)$ is the approximated function. The $\mathrm{RE}$ of (9) is too high and cannot be plotted in the same figure.

\section{INTEGER POWERS OF THE $Q$-Function}

The expression in (6) can be also applied to derive efficient approximative values for the integer powers of the Gaussian $Q$-function, i.e.

$$
Q^{n}(x) \approx \frac{1}{2^{n}} f^{n}\left(\frac{x}{\sqrt{2}}, A, B\right)
$$


which can be written after the binomial expansion as

$$
\left.Q^{n}(x) \approx \frac{1}{2^{n}} \sum_{k=0}^{n}\left(\begin{array}{l}
n \\
k
\end{array}\right)(-1)^{n-k} e^{-A(n-k) \frac{x}{\sqrt{2}}}\right) \frac{e^{-A n \frac{x^{2}}{\sqrt{2}}}}{(B \sqrt{\pi} x)^{n}}
$$

where $\left(\begin{array}{l}n \\ k\end{array}\right)$ denotes the binomial coefficient.

\section{A. Evaluation of the ASEP of Differentially Encoded QPSK in Nakagami-m fading}

The integer powers of the Gaussian $Q$-function are involved in several communications problems, including the evaluation of the error probability in AWGN, and the ASEP of coherent detection of 4-ary orthogonal signaling and DE-QPSK over fading channels. Simon, in his pioneering work [8], derived the Craig-type forms up to fourth power of the Gaussian $Q$ function and applied them to evaluate the ASEP of DE-QPSK in fading channels. However, for Nakagami- $m$ fading, the final formula involves definite integrals and is valid for integer values of the $m$ parameter $[9,(8.124)]$.

Next, we apply the approximate expression in (12) to evaluate in closed-form the ASEP of the DE-QPSK in Nakagami$m$ fading. The SNR per symbol, $\gamma$, is distributed according to gamma distribution with a probability density function (pdf) given by $[9,(2.21)]$

$$
p_{\gamma}(\gamma)=\frac{m^{m} \gamma^{m-1}}{\bar{\gamma}^{m} \Gamma(m)} e^{-\frac{m \gamma}{\bar{\gamma}}}
$$

where $m$ is the fading parameter, which ranges from 0.5 to $\infty, \bar{\gamma}$ is the average SNR per symbol and $\Gamma(\cdot)$ denotes the gamma function [7]. The SEP of DE-QPSK on the AWGN channel is given by [8]

$$
P_{s}(E)=4 Q(\sqrt{\gamma})-8 Q^{2}(\sqrt{\gamma})+8 Q^{3}(\sqrt{\gamma})-4 Q^{4}(\sqrt{\gamma})
$$

The ASEP is obtained by averaging (14) over $p_{\gamma}(\gamma)$, i.e.

$$
\bar{P}_{s}(E)=\int_{0}^{\infty} P_{s}(E) p_{\gamma}(\gamma) d \gamma
$$

By substituting (12) in (15), integrals of the form

$$
I_{1}=\int_{0}^{\infty} x^{c} e^{-a x} d x
$$

and

$$
I_{2}=\int_{0}^{\infty} e^{-a x} e^{-b \sqrt{x}} x^{c} d x
$$

arise, where $a>0, b>0, c>-1$, which implies that $m>2$. These integrals can be evaluated using $[6,3.351 .3]$ and $[6$, $3.462 .1]$ as

$$
I_{1}=a^{-c-1} \Gamma(c+1)
$$

and

$$
\begin{aligned}
I_{2}=a^{-\frac{3}{2}-c}\left[\sqrt{a} \Gamma(c+1){ }_{1} F_{1}\left(c+1 ; \frac{1}{2} ; \frac{b^{2}}{4 a}\right)-\right. \\
\left.b \Gamma\left(c+\frac{3}{2}\right){ }_{1} F_{1}\left(c+\frac{3}{2} ; \frac{3}{2} ; \frac{b^{2}}{4 a}\right)\right]
\end{aligned}
$$

where ${ }_{1} F_{1}(\cdot ; \cdot ; \cdot)$ denotes the confluent hypergeometric function [6, 9.21], finally resulting in a closed-form solution for $\bar{P}_{s}(E)$ which is omitted here due to space limitations.
TABLE II

THE COMPARISON OF THE EXACT AND APPROXIMATED ASEP OF THE DEFERENTIALLY ENCODED QPSK

$m=2.5$

\begin{tabular}{|l|l|l|l|l|}
\hline $\bar{\gamma}$ & Exact & Chiani et al. & Börjesson et al. & $(6)$ \\
\hline 0 & 0.481372 & 0.506094 & 0.400987 & 0.484639 \\
\hline 5 & 0.213309 & 0.243585 & 0.235053 & 0.213539 \\
\hline 10 & 0.0432554 & 0.0512244 & 0.0727512 & 0.0428847 \\
\hline 15 & 0.00440965 & 0.00525679 & 0.0104954 & 0.00434423 \\
\hline 20 & 0.000309771 & 0.000369446 & 0.000877668 & 0.000304364 \\
\hline 25 & 0.000018772 & 0.000022386 & 0.000056820 & 0.000018427 \\
\hline \multicolumn{5}{|c|}{$m=3.5$} \\
\hline 0 & 0.476344 & 0.504489 & 0.399072 & 0.479782 \\
\hline 5 & 0.194979 & 0.226913 & 0.226411 & 0.194906 \\
\hline 10 & 0.0288924 & 0.0347995 & 0.0597309 & 0.0284718 \\
\hline 15 & 0.00150192 & 0.0018022 & 0.00544383 & 0.00146504 \\
\hline 20 & 0.000040809 & 0.000048692 & 0.00020369 & 0.000039628 \\
\hline 25 & $8.39755 \mathrm{e}-7$ & $9.99544 \mathrm{e}-7$ & $4.76981 \mathrm{e}-6$ & $8.14168 \mathrm{e}-7$ \\
\hline
\end{tabular}

\section{B. Numerical Results and Discussion}

In Table II, we compare the exact ASEP of the DEQPSK, calculated by numerical integration using (15) with the approximated ones using (12) and the corresponding ones in (8), (9) and (6). It is observed that (12) results in a tighter approximation of the ASEP over the whole range of the SNR values.

We should note here that as mentioned in [8], although for the AWGN channel the first two terms of (14) are typically sufficient for a good approximation of the error performance in high SNR applications, in fading channel all four terms are important because of the need to average over the SNR between zero and infinity. This fact strengthens the argument that (6) can be efficiently applied for the evaluation of error probabilities in fading channels, since a declination from the exact value would lead in approximation errors, especially in the low SNR region.

\section{REFERENCES}

[1] M. K. Simon, Probability Distributions Involving Gaussian Random Variables: A Handbook for Engineers and Scientists. Boston, MA: Kluwer, 2002.

[2] N. C. Beaulieu, "A simple series for personal computer computation of the error function Q(·)," IEEE Trans. Commun., vol. 37, pp. 989-991, Sept. 1989.

[3] C. Tellambura and A. Annamalai, "Efficient computation of erfc(x) for large arguments," IEEE Trans. Commun., vol. 48, pp. 529-532, Apr. 2000.

[4] P. O. Börjesson and C. E. Sundberg, "Simple approximations of the error function $\mathrm{Q}(\mathrm{x})$ for communications applications," IEEE Trans. Commun., vol. COM-27, pp. 639-643, Mar. 1979.

[5] M. Chiani, D. Dardari, and M. K. Simon, "New exponential bounds and approximations for the computation of error probability in fading channels," IEEE Trans. Wireless Commun., vol. 2, pp. 840-845, July 2003.

[6] I. S. Gradshteyn and I. M. Ryzhik, Tables of Integrals, Series, and Products, 5th ed. San Diego, CA: Academic, 1994.

[7] M. Abramovitz and I. A. Stegun, Handbook of Mathematical Functions. New York: Dover, 1972.

[8] M. K. Simon, "Integral representations of certain integer powers of the Gaussian Q-function and their application," IEEE Commun. Lett., vol. 6, pp. 532-534, Dec. 2002.

[9] M. K. Simon and M. Alouini, Digital Communication over Fading Channels, 2nd ed. John Wiley, 2005. 